\title{
Quasi-molecular lines in Lyman wings of cool DA white dwarfs
}

\section{Application to FUSE observations of G 231-40}

\author{
G. Hébrard ${ }^{1}$, N. F. Allard ${ }^{1,2}$, I. Hubeny ${ }^{3}$, S. Lacour ${ }^{4,1}$, R. Ferlet ${ }^{1}$, and A. Vidal-Madjar ${ }^{1}$ \\ 1 Institut d'Astrophysique de Paris, CNRS, 98bis boulevard Arago, 75014 Paris, France \\ 2 Observatoire de Paris-Meudon, LERMA, 92195 Meudon Principal Cedex, France \\ 3 NOAO, 950 North Cherry Avenue, Tucson, AZ 85726, USA \\ 4 Department of Physics and Astronomy, Johns Hopkins University, Baltimore, MD 21218, USA
}

Received 3 April 2002 / Accepted 5 August 2002

\begin{abstract}
We present new theoretical calculations of the total line profiles of Lyman $\alpha$ and Lyman $\beta$ which include perturbations by both neutral hydrogen and protons and all possible quasi-molecular states of $\mathrm{H}_{2}$ and $\mathrm{H}_{2}^{+}$. They are used to improve theoretical modeling of synthetic spectra for cool DA white dwarfs. We compare them with FUSE observation of G231-40. The appearance of the line wings between Lyman $\alpha$ and Lyman $\beta$ is shown to be sensitive to the relative abundance of hydrogen ions and neutral atoms, and thereby to provide a temperature diagnostic for stellar atmospheres and laboratory plasmas.
\end{abstract}

Key words. line: profiles - radiation mechanisms: general - stars: atmospheres - stars: individual: G231-40 - white dwarfs ultraviolet: stars

\section{Introduction}

Structures in the Lyman $\alpha$ and Lyman $\beta$ line wings have been identified with free-free transitions which take place during binary close collisions of the radiating $\mathrm{H}$ atom and a perturbing atom or ion (Allard et al. 1998a, 1998b, 1999). The characteristics of these features (position, amplitude, and shape), due to the formation of quasi-molecules during collisions between the radiating atom and perturbers, depend directly on the potential energy curves correlated to the atomic levels of the transition (Allard \& Kielkopf 1982).

Two satellite absorption features at $1058 \AA$ and $1076 \AA$ due to collisions of atomic hydrogen with protons were first identified in the spectrum of the DA white dwarf Wolf 1346, as observed with the Hopkins Ultraviolet Telescope (Koester et al. 1996). These satellites in the red wing of Lyman $\beta$ are in the Far Ultraviolet Spectroscopic Explorer (FUSE) spectral range (Moos et al. 2000); furthermore, Lyman $\beta$ profiles are also the subject of an ongoing study of the far ultraviolet spectrum of dense hydrogen plasmas.

In Allard et al. (1998a) we presented theoretical profiles of Lyman $\beta$ perturbed solely by protons. The calculations were based on the accurate theoretical $\mathrm{H}_{2}^{+}$molecular potentials of Madsen \& Peek (1971) to describe the interaction between radiator and perturber, and dipole transition moments of Ramaker \& Peek (1972). The line profiles were included as a

Send offprint requests to: G. Hébrard, e-mail: hebrard@iap.fr source of opacity in model atmospheres for hot white dwarfs, and the predicted spectra compared well with the observed ORFEUS and FUSE spectra (Koester et al. 1998; Wolff et al. 2001).

Ab initio calculations of Drira (1999) of electronic transition moments for excited states of the $\mathrm{H}_{2}$ molecule and molecular potentials of Detmer et al. (1998) allowed us to compute Lyman $\beta$ profiles perturbed by neutral atomic hydrogen (Allard et al. 2000). The appearance of a broad satellite situated at $1150 \AA$ makes necessary to take into account the total contribution of both the Lyman $\alpha$ and Lyman $\beta$ wings of H perturbed simultaneously by neutrals and protons.

We show that the shape of the wings in the region between Lyman $\beta$ and Lyman $\alpha$ is particularly sensitive to the relative abundance of the neutral and ion perturbers responsible for the broadening of the lines.

These new profiles have been used to predict synthetic spectra for cool DA white dwarfs which present structures at $1600 \AA$ and $1400 \AA$ in the Lyman $\alpha$ wing due respectively to quasi-molecular absorption of the $\mathrm{H}_{2}$ and $\mathrm{H}_{2}^{+}$molecules. These last two structures have been demonstrated to be a very sensitive temperature indicators in DA white dwarfs. The relative strength of these two satellite features depends very strongly on the degree of ionization in the stellar atmosphere, and thus on the stellar parameters $T_{\text {eff }}$ and $\log g$ (Koester \& Allard 1993; Koester et al. 1994; Bergeron et al. 1995). 


\section{Theoretical line profiles}

\subsection{Theory}

We use a general unified theory in which the electric dipole moment varies during a collision; a detailed description of the theory as applied to the shape of the Lyman lines has been given by Allard et al. (1999). The obtained line profiles fit the spectra of laser-produced hydrogen plasmas (Kielkopf \& Allard 1998).

Our approach requires prior knowledge of accurate theoretical molecular potentials to describe the interaction between radiator and perturber, and knowledge of the variation of the radiative dipole moment with atom-atom and atom-ion separation for each molecular state. This effect is important when the dipole moment varies in the region of inter-nuclear distance where the satellite is formed, and thus cannot be neglected. In the case of Lyman $\beta$ satellites, due to $\mathrm{H}-\mathrm{H}^{+}$collisions, we have shown that large changes (up to $60 \%$ ) in the intensity of the satellites may occur when the variation of the dipole moment is taken into account (Allard et al. 1998a). This result is also valid for other lines; it increases by a factor of about 2 the main satellites of Lyman $\alpha$ (Allard et al. 1999). Previous line profile calculations using constant dipole moment have been used to interpret IUE (International Ultraviolet Explorer) and Hubble Space Telescope spectra by Koester \& Allard (1993), Koester et al. (1994), and Bergeron et al. (1995). The synthetic spectra presented here used improved Lyman $\alpha$ line profiles of Allard et al. (1998b) which have been already included in stellar atmosphere programs for the computation of stellar atmosphere model and synthetic spectra of $\lambda$ Bootis stars. A comparison of these calculations with observations made with the IUE demonstrated that these last improvements are of fundamental importance for obtaining a better quantitative interpretation of the spectra and for determining stellar atmospheric parameters (Allard et al. 1998b).

\subsection{Lyman $\beta$ in $H-H$ collisions}

The Lyman profiles and satellites are calculated at the low densities met in the atmospheres of stars. The typical particle densities $\left(10^{15}\right.$ to $\left.10^{17} \mathrm{~cm}^{-3}\right)$ allows us to use an expansion of the autocorrelation function in powers of density as described in Allard et al. (1994) and Royer (1971). Line profiles are normalized so that over $1 / \lambda=\omega\left(\mathrm{cm}^{-1}\right)$ they integrate to 1 .

The only line feature of the Lyman $\beta$ profile is a broad absorption line satellite situated at $1150 \AA$ due to the $B^{\prime \prime} \bar{B}^{1} \Sigma_{u}^{+}-$ $\mathrm{X}^{1} \Sigma_{\mathrm{g}}^{+}$molecular transition of $\mathrm{H}_{2}$ (Fig. 1). The recent ab initio calculations of Spielfiedel (2001, private communication) have shown that for the isolated radiating atom $(R \rightarrow \infty)$ this transition is not asymptotically forbidden as it was explicitly stated in Allard et al. (2000).

The line satellite shown in Fig. 1 presents a shoulder at $1120 \AA$, a similar shape has been obtained for the $1600 \AA$ satellite. In Fig. 6 of Allard et al. (1999) both theory and experiment show an oscillatory structure between the satellite and the line, with a minimum at about $1525 \AA$. These oscillations are an interference effect (Royer 1971; Sando \& Wormhoudt 1973), and

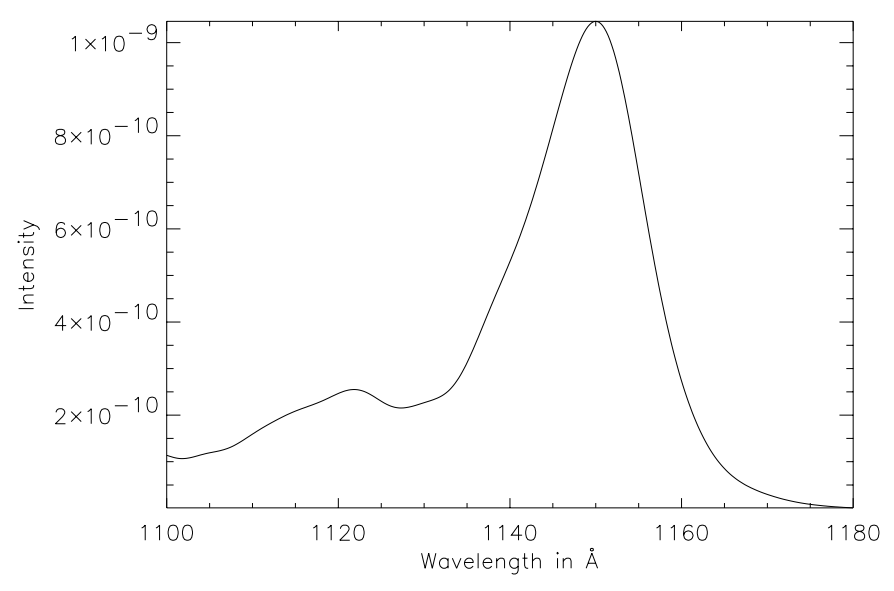

Fig. 1. Lyman $\beta$ satellite due to $\mathrm{H}-\mathrm{H}$ collisions. The neutral density $n_{\mathrm{H}}$ is $1 \times 10^{16} \mathrm{~cm}^{-3}$.

are expected to depend on the relative velocity of the collision and therefore on temperature.

This satellite of Lyman $\beta$ is quite far from the unperturbed Lyman $\beta$ line center, actually closer to the Lyman $\alpha$ line. It is therefore necessary to take into account the total contribution of both the Lyman $\alpha$ and Lyman $\beta$ wings of $\mathrm{H}$ perturbed simultaneously by neutrals and protons and to study the variation of this part of the Lyman series with the relative density of ionized and neutral atoms.

\subsection{Lyman $\beta$ in $\mathrm{H}-\mathrm{H}$ and $\mathrm{H}-\mathrm{H}^{+}$collisions}

In Allard et al. (1998a) we presented Lyman $\beta$ profiles perturbed by protons. The line profile calculations were done without using the expansion in density and then were valid from the center to the far wing and allowed a comparison of the amplitudes of the satellites to the line core. The profiles at different densities of $\mathrm{H}^{+}$were displayed in Fig. 8.

In Fig. 2 we show the sum of the profiles of Lyman $\alpha$ and Lyman $\beta$ perturbed by collisions with neutral hydrogen and protons for different densities $n_{\mathrm{H}}$.

We can see that a ratio of 5 between the neutral and proton density is enough to make the quasi-molecular $\mathrm{H}_{2}$ satellite appear in the far wing. Note that the $1120 \AA$ shoulder of the satellite is still visible in the total profile.

\section{Synthetic spectra for DA white dwarfs}

We now take into account these absorption features of the $\mathrm{H}_{2}^{+}$ and $\mathrm{H}_{2}$ quasi-molecules to calculate synthetic spectra for DA white dwarfs. Atmosphere models and synthetic spectra have been calculated using the computer programs TLUSTY and SYNSPEC (Hubeny 1988; Hubeny \& Lanz 1992, 1995). The LTE model atmospheres assume a pure hydrogen composition including the quasi-molecular opacities.

Below $T_{\text {eff }}=15000 \mathrm{~K}$, the atmosphere of DA white dwarfs become convective, and model atmospheres for these stars are usually calculated using several variants of the standard mixing-length theory. According Bergeron et al. (1992), we used the so-called ML2 prescription with $\alpha=0.6$, which 


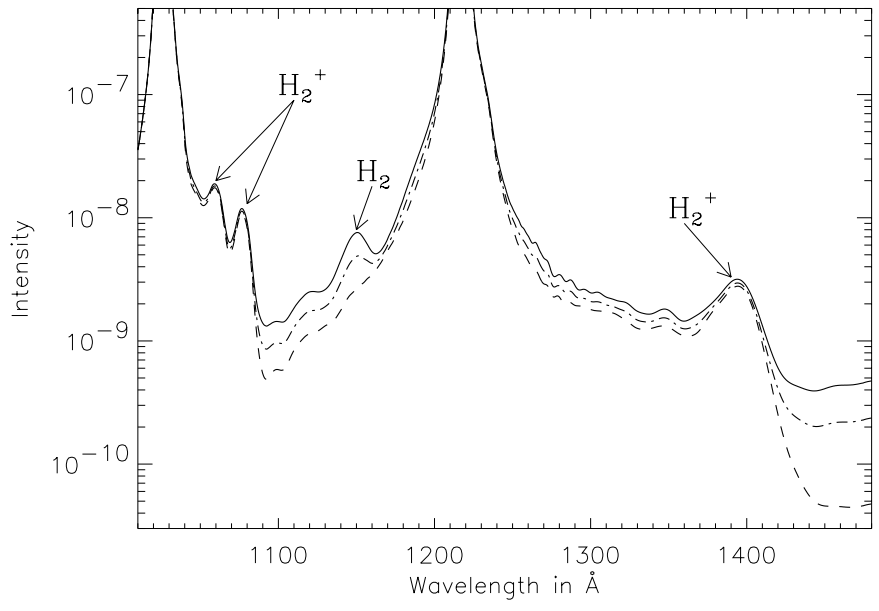

Fig. 2. Total profile of Lyman $\alpha$ and Lyman $\beta$ perturbed by neutral hydrogen and protons. Three different neutral densities $\left(1 \times 10^{17}, 5 \times\right.$ $10^{16}, 1 \times 10^{16} \mathrm{~cm}^{-3}$, top to the bottom) are compared for a fixed ion density $\left(1 \times 10^{16} \mathrm{~cm}^{-3}\right)$.

was shown to provide excellent internal consistency between ultraviolet and optical temperatures.

The temperature range where both $\mathrm{H}_{2}$ and $\mathrm{H}_{2}^{+}$Lyman $\beta$ satellites are visible is relatively small; roughly between 14500 to $11000 \mathrm{~K}$ (Fig. 3, upper panel). This dependence of the theoretical profiles on temperature is extremely strong because of the relative importance of perturbations by neutral versus ionized hydrogen. The satellite appearance is then very sensitive to the degree of ionization and may be used as a temperature diagnostic.

In order to see all the Lyman line satellites, synthetic spectra are plotted from 1020 to $1800 \AA$ (Fig. 3, lower panel). Below $T_{\text {eff }}=13000 \mathrm{~K}$ the $\mathrm{H}_{2}^{+}$starts to disappear but the broad $\mathrm{H}_{2}$ satellite at $1150 \AA$ is still present and the one at $1600 \AA$ slowly increases.

\section{FUSE observations of G 231-40}

\subsection{Observations and data reduction}

G 231-40 (WD $2117+539$ ) is a cool DA white dwarf located at Galactic coordinates $l=95.0^{\circ}$ and $b=-3.3^{\circ}$; it is a relatively bright white dwarf: $V=12.3$. This target was observed with FUSE as part of the French Guaranteed Time Observing Programs (program Q210). Two exposures were obtained on 2001 July 3 in time-tagged photon address mode (TTAG) with the object in the large aperture (LWRS). The total duration was $\sim 1.6 \mathrm{~h}$ (see Table 1). Details of the FUSE instrument may be found in Moos et al. (2000) and Sahnow et al. (2000).

The one-dimensional spectra were extracted from the twodimensional detector images and calibrated using version 2.0.5 of the CalFUSE pipeline. The eight FUSE detector segments of the two exposures (i.e. 16 spectra) were co-added and projected on a $0.16 \AA$-pixel base, i.e. pixels about 25 times larger than the original FUSE detectors pixels. This degradation of the FUSE spectral resolution (typically $\lambda / \Delta \lambda \simeq 15000$ for this kind of target in the large slit; see Hébrard et al. 2002; Wood et al. 2002) is of no effect on the shapes of the large stellar features which we study and allows us to increase the signal-to-noise ratio. At this resolution, no spectral shifts were detected between the 16 different co-added spectra.

Poor quality edge of each segment were not included in the sum. We used the following spectral ranges for each segment: 1010-1085 $\AA$ (SiC1A), 910-992.5 $\AA$ (SiC1B), 922-1005 $\AA$ (SiC2A), 1016-1101 ̊ (SiC2B), 1004-1081.5 (LiF1A), 1096-1187 $\AA$ (LiF1B), 1087-1181 $\AA$ (LiF2A), and 1012-1073 $\AA$ (LiF2B). As the LiF1B segment presents a known large-scale distortion ("the worm", D. Sahnow 2000, private communication) in the flux calibration around 1120-1170 A (see, e.g., Hébrard et al. 2002), we normalized the large-scale shape of the LiF1B segment to the shape of the LiF2A segment, using a polynomial fit. We also did not include the part of the LiF2A spectra in the ranges 1134.6-1135.6 $\AA$ and $1151.5-1153 \AA$ because they were deteriorated by the socalled "walk" problem, which is a distortion in the FUSE spectra at airglow wavelengths ( $\lambda 1134 \AA \mathrm{N}_{\mathrm{I}}$ and $\lambda 1152 \AA \mathrm{O}_{\mathrm{I}}$ lines in the present case) caused by variation of position with pulse height (Sahnow 2001, private communication). The final FUSE spectrum is plotted in Fig. 4. Note that the interstellar $\mathrm{N}_{\mathrm{I}} \lambda 1134 \AA$ triplet is detected on this line of sight.

Since 2001, repeated observations of standard white dwarfs indicate a slow degradation in the effective area of the FUSE spectrograph. The decline affects the flux up to $20 \%$, and that mainly for the LiF2A channel. A dime-dependent flux correction is in development for a future version of the pipeline, but is not included in the CalFUSE version 2.0.5.

In order to complement the 910-1187 A FUSE spectrum, we retrieved two IUE low dispersion spectra of G 231-40 from the STScI MAST archive. These spectra were obtained in March 1983 and they were reduced using the NEWSIPS processing (see Table 1). They yield the spectral coverage 1150-1980 A at a resolution of about $7 \AA$ (Wegner 1984). The flux calibration of the FUSE and the IUE spectra are in good agreement (see Fig. 4, lower panel).

\subsection{Comparison with theoretical spectra}

As a change in temperature can be compensated by a change in surface gravity we have used $\log g$ determined from optical analysis. Holberg et al. (1998) give $(14490 ; 7.85)$ for $\left(T_{\mathrm{eff}}\right.$; $\log g$ ), the atmospheric parameters being determined from spectroscopic observations by Bergeron et al. (1992). Model atmospheres and synthetic spectra were calculated in the range 15500 to $14500 \mathrm{~K}$ by steps of $100 \mathrm{~K}$, using $\log g=7.85$.

$\mathrm{H}^{-}$opacity (Wishart 1979) has been added in the atmosphere model calculation and gives a feature at $1130 \AA$ also present in the FUSE observation (see Fig. 4).

Figure 4, upper panel, shows the comparison of the FUSE observation with our best fit obtained for $T_{\text {eff }}=14800 \mathrm{~K}$. The decreasing of sensitivity on the segment LiF2A (Sect. 4.1) is the main explanation of the difference between the data and the model between 1120 and $1180 \AA$. The $\mathrm{H}_{2}$ satellite $(1150 \AA)$ is no more visible, as expected, in this range of temperature.

Figure 4 shows a good agreement between the FUSE and $I U E$ observations of Lyman lines and predicted spectra over the 


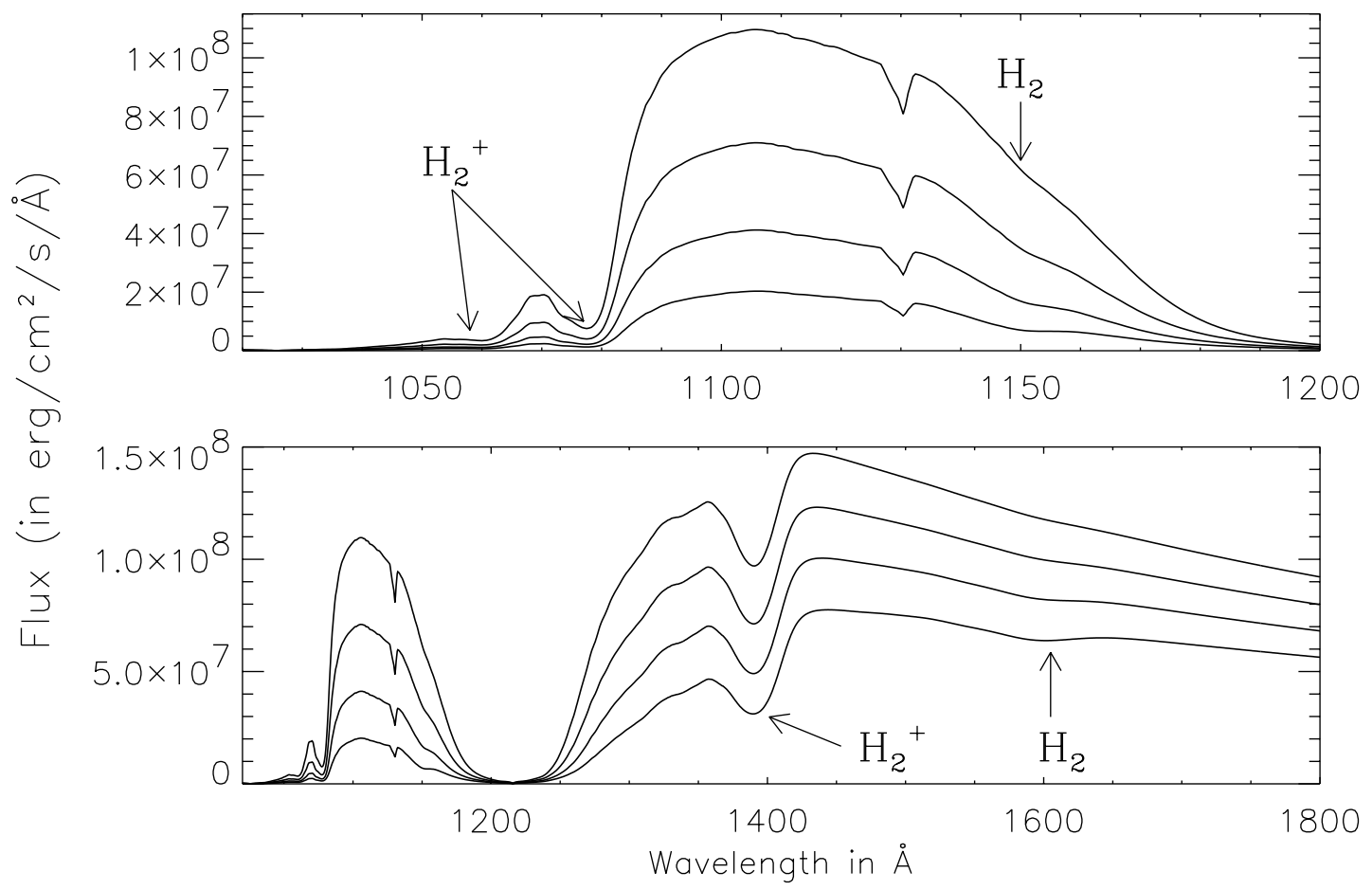

Fig. 3. Synthetic spectra for DA white dwarfs including Lyman $\alpha$ and Lyman $\beta$ satellites, on spectral ranges 1020-1200 $\AA$ (upper panel) and $1020-1800 \AA$ (lower panel). $T_{\text {eff }}=14500,14000,13500,13000 \mathrm{~K}$ (from top to bottom) and $\log g=8.0$. The flux, at the stellar surface, is integrated over angles.
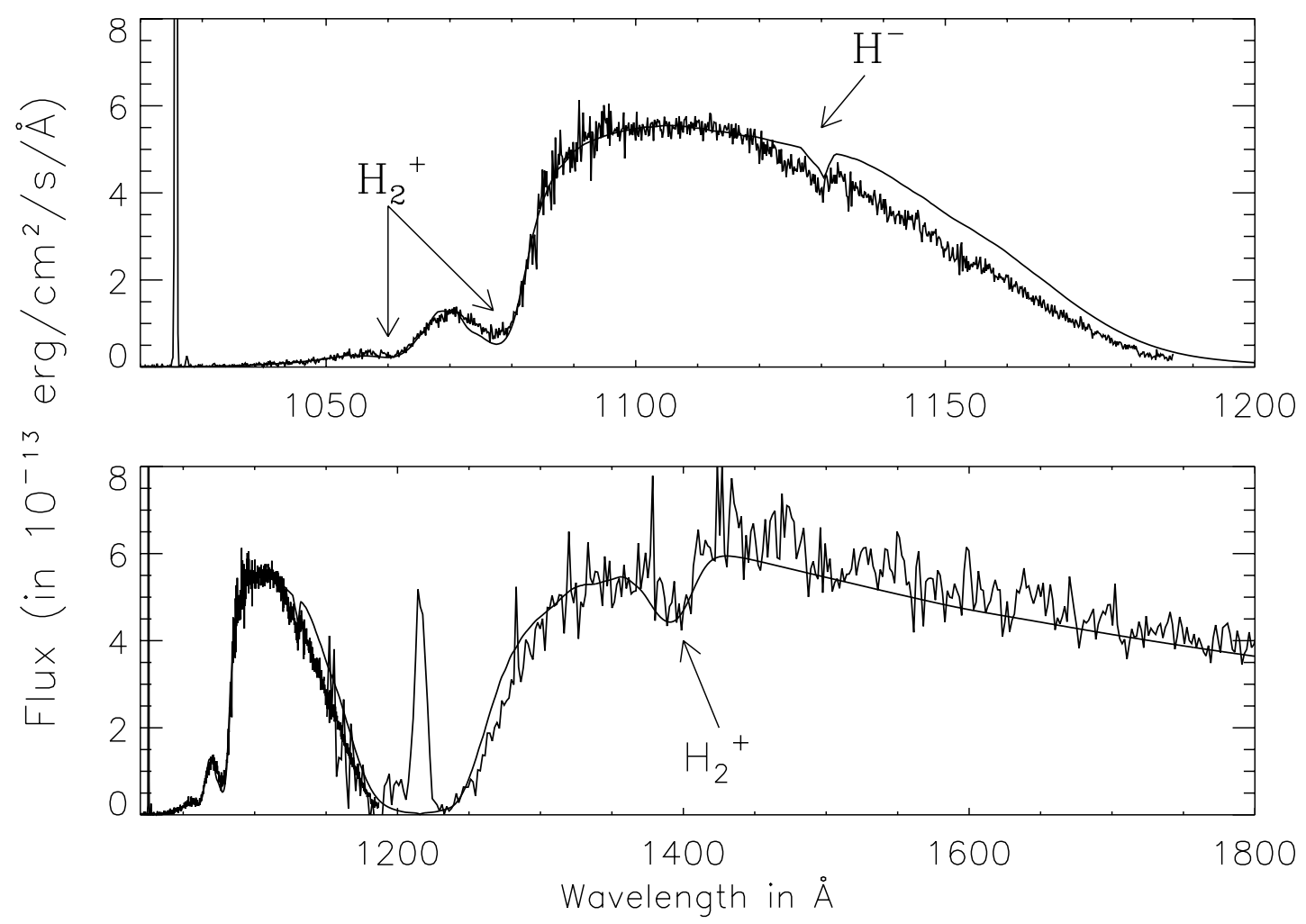

Fig. 4. FUSE (1020-1187 ̊, upper and lower panels) and IUE (1150-1800 ̊, lower panel) spectra of G 231-40 compared with a theoretical model for $T_{\text {eff }}=14800 \mathrm{~K}$ and $\log g=7.85$. Emissions at Lyman $\alpha$, Lyman $\beta$, and $1027.5 \AA$ are due to H I and O I airglow. The difference between the data and the model between 1120 and $1180 \AA$ is mainly due to an uncorrected decreasing of sensitivity on the FUSE segment LiF2A (see Sect. 4.1). Notice the $\mathrm{H}^{-}$feature at $1130 \AA$. 
Table 1. Observation log.

\begin{tabular}{cccccc}
\hline \hline Instrument & Observation & Date of observation & Aperture & Exposure time $(\mathrm{s})$ & Spectral extraction \\
\hline FUSE & Q2100101001 & 2001 July 3 & LWRS $\left(30^{\prime \prime} \times 30^{\prime \prime}\right)$ & 2940 & CalFUSE 2.0.5 \\
FUSE & Q2100101002 & 2001 July 3 & LWRS $\left(30^{\prime \prime} \times 30^{\prime \prime}\right)$ & 2864 & CalFUSE 2.0.5 \\
$I U E$ & SWP19532 & 1983 March 24 & SWLA $\left(10^{\prime \prime} \times 20^{\prime \prime}\right)$ & 720 & NEWSIPS \\
$I U E$ & SWP19533 & 1983 March 24 & SWLA $\left(10^{\prime \prime} \times 20^{\prime \prime}\right)$ & 720 & NEWSIPS \\
\hline
\end{tabular}

whole range from 1020 to $1800 \AA$ using atmospheric parameters determined from the optical range.

The feature near $\lambda 995 \AA$ due to a Lyman $\gamma$ satellite, which is visible in the spectra of hotter objects, is no more present in the temperature range of $\mathrm{G} 231-40$ (the flux of G 231-40 is below $1 \times 10^{-14} \mathrm{erg} \mathrm{cm}^{-2} \mathrm{~s}^{-1} \AA^{-1}$ at $995 \AA$ ). It was visible in FUSE observations of CD $-38^{\circ} 10980$ (Wolff et al. 2001), $H U T$ spectrum of Wolf 1346 (Koester et al. 1996), and in some ORFEUS spectra (Koester et al. 1998). It was also recently seen in FUSE spectra of Sirius B (Holberg et al. 2002). This feature, due to a Lyman $\gamma$ satellite, is now included in our calculations. We have shown that a larger temperature is required to get it detectable (Allard et al. 2002).

\section{Conclusion}

From theoretical profiles including the new Lyman $\beta$ opacities it can be predicted that Lyman $\beta$ satellites should be detectable roughly between $25000 \mathrm{~K}$ to $11000 \mathrm{~K}$. Within this range in a very small domain of temperatures, the whole profile should present $\mathrm{H}_{2}$ and $\mathrm{H}_{2}^{+}$satellites of the Lyman $\alpha$ and Lyman $\beta$ lines. FUSE observations of cooler white dwarfs in the ZZ Ceti range stars would offer the best opportunity to determine accurate stellar parameters as $T_{\text {eff }}$ and $\log g$ for these stars.

Acknowledgements. This work is based on data obtained for the French Guaranteed Time by the NASA-CNES-CSA FUSE mission operated by the Johns Hopkins University. I.H. would like to thank the PNPS (Programme National de Physique Stellaire) for travel support to IAP where part of this work was done. We would like to thank Jean Dupuis for useful comments on this work.

\section{References}

Allard, N. F., \& Kielkopf, J. 1982, Rev. Mod. Phys., 54, 1103

Allard, N. F., Koester, D., Feautrier, N., \& Spielfiedel, A. 1994, A\&A, 200,58

Allard, N. F., Kielkopf, J. F., \& Feautrier, N. 1998a, A\&A, 330, 782
Allard, N. F., Drira, I., Gerbaldi, M., Kielkopf, J. F., \& Spielfiedel, A. 1998b, A\&A, 335, 1124

Allard, N. F., Royer, A., Kielkopf, J. F., \& Feautrier, N. 1999, Phys. Rev. A, 60, 1021

Allard, N. F., Kielkopf, J. F., Drira, I., \& Schmelcher, P. 2000, Eur. Phys. J. D, 12, 263

Allard, N. F., Bourdreux, S., Kielkopf, J. F., et al. 2002, 13th European Workshop on White Dwarfs, Napoli, 24-28 juin 2002

Bergeron, P., Saffer, R., \& Liebert, J. 1992, ApJ, 394, 228

Bergeron, P., Wesemael, F., Lamontagne, R., et al. 1995, ApJ, 449, 258

Detmer, T., Schmelcher, P., \& Cederbaum, L. S. 1998, J. Chem. Phys., 109, 9694

Drira, I. 1999, J. Mol. Spectroscopy, 198, 52

Hébrard, G., Lemoine, M., Vidal-Madjar, A., et al. 2002, ApJS, 140, 103

Holberg, J. B., Barstow, M. A., \& Sion, E. M. 1998, ApJS, 119, 207

Holberg, J. B., Kruk, J. W., Barstow, M. A., et al. 2002, FUSE Science and Data Workshop, JHU, Baltimore, 56

Hubeny, I. 1988, Comp. Phys. Comm., 52, 103

Hubeny, I., \& Lanz, T. 1992, A\&A, 262, 501

Hubeny, I., \& Lanz, T. 1995, ApJ, 439, 875

Kielkopf, J. F., \& Allard, N. F. 1995, ApJ, 450, L75

Kielkopf, J. F., \& Allard, N. F. 1998, Phys. Rev. A, 58, 4416

Koester, D., \& Allard, N. F. 1993, in White Dwarfs: Advances in Observation and Theory, ed. M. Barstow (Kluwer: Dordrecht), 237

Koester, D., Allard, N. F., \& Vauclair, G. 1994, A\&A, 291, L9

Koester, D., Finley, D. S., Allard, N. F., Kruk, J. W., \& Kimble, R. A. 1996, ApJ, 463, L93

Koester, D., Sperhake, U., Allard, N. F., Finley, D. S., \& Jordan, S. 1998, A\&A, 336, 276

Madsen, M. M., \& Peek, J. M. 1971, Atomic Data, 2, 171

Moos, H. W., Cash, W. C., Cowie, L. L., et al. 2000, ApJ, 538, L1

Ramaker, D. E., \& Peek, J. M. 1972, J. Phys. B, 5, 2175

Royer, A. 1971, Phys. Rev. A, 43, 499

Sahnow, D. J., Moos, H. W., Ake, T. B., et al. 2000, ApJ, 538, L7

Sando, K. M., \& Wormhoudt, J. G. 1973, Phys. Rev. A, 7, 1889

Wishart, A. W. 1979, MNRAS, 187, 59

Wegner, G. 1984, AJ, 89, 1050

Wolff, B., Kruk, J. W., Koester, D., et al. 2001, A\&A, 373, 674

Wood, B. E., Linsky, J. L., Hébrard, G., et al. 2002, ApJS, 140, 91 